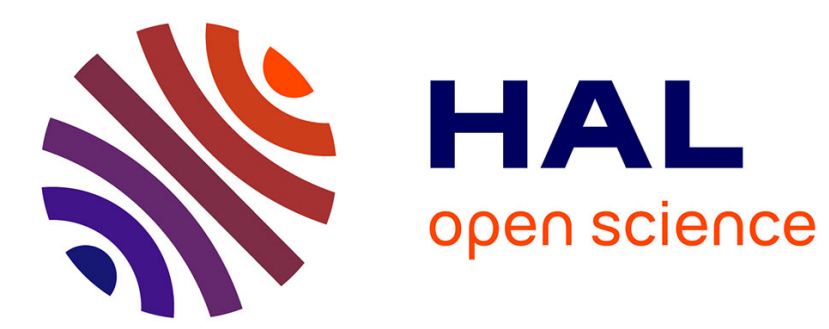

\title{
PAROIS DANS LES FILMS MINCES
}

M. Louis Néel

\section{To cite this version:}

M. Louis Néel. PAROIS DANS LES FILMS MINCES. Journal de Physique Colloques, 1968, 29 (C2), pp.C2-87-C2-94. 10.1051/jphyscol:1968215 . jpa-00213530

\section{HAL Id: jpa-00213530 https://hal.science/jpa-00213530}

Submitted on 1 Jan 1968

HAL is a multi-disciplinary open access archive for the deposit and dissemination of scientific research documents, whether they are published or not. The documents may come from teaching and research institutions in France or abroad, or from public or private research centers.
L'archive ouverte pluridisciplinaire HAL, est destinée au dépôt et à la diffusion de documents scientifiques de niveau recherche, publiés ou non, émanant des établissements d'enseignement et de recherche français ou étrangers, des laboratoires publics ou privés. 


\title{
PAROIS DANS LES FILMS MINCES
}

\author{
par M. Louis NéEL \\ Laboratoire d'Electrostatique et de Physique du Métal, C.N.R.S., Grenoble.
}

\begin{abstract}
Résumé. - Rappel des principaux résultats théoriques et expérimentaux acquis au cours des dernières années sur les parois dans les couches minces, passage des parois de Bloch aux parois de Néel, avec les "cross-tie) comme intermédiaire. On expose ensuite brièvement les résultats obtenus sur les parois et quasi-parois de Bloch et de Néel dans les couches minces multiples et on souligne la difficulté d'obtenir des résultats théoriques précis, malgré la simplicité apparente du problème.
\end{abstract}

\begin{abstract}
Review of the principal theoretical and experimental results obtained in the past years on domain walls in thin films; transition of the Bloch walls to Néel walls with the «cross-tie» as the intermediary. One shows briefly, the results obtained on Bloch and Néel walls and quasi-walls in multiple thin films and emphasizes the difficulties in obtaining precise theoretical results, despite the apparent simplicity of the proble.n.
\end{abstract}

1. Parois de Bloch dans les substances massives. J'ai simplement l'intention au cours de cet exposé d'introduction, destiné à de non-spécialistes, de rappeler les principaux résultats acquis aujourd'hui sur les particularités que présentent dans les films minces les parois de séparation entre les domaines élémentaires de Weiss. On sait depuis F. Bloch qu'une paroi séparant deux domaines, par exemple à aimantations antiparallèles $+\mathbf{J}_{\mathrm{s}}$ et $-\mathbf{J}_{s}$, n'est pas un plan géométrique mais un mur d'épaisseur finie à l'intérieur duquel la dircction de l'aimantation spontanée tourne d'une manière continue de $+\mathbf{J}_{\mathrm{s}} \grave{a}-\mathbf{J}_{\mathrm{s}}$.

L'énergie de la paroi est la somme de l'énergie d'échange

$$
F_{\mathrm{w}}=A\left[(\nabla \alpha)^{2}+(\nabla \beta)^{2}+(\nabla \gamma)^{2}\right]
$$

et de l'énergie d'anisotropie uniaxiale

$$
F_{\mathrm{c}}=K \alpha^{2}
$$

$\left(\alpha, \beta, \gamma\right.$ cosinus directeurs de $\mathbf{J}_{\mathrm{s}}, \alpha$ correspondant à la direction de facile aimantation). Comme $\nabla \alpha$ est de l'ordre de $1 / a$, l'énergie d'échange d'une paroi d'épaisseur $a$ est de l'ordre $A / a$ par $\mathrm{cm}^{2}$; elle est inversement proportionnelle à l'épaisseur de la paroi. L'énergie d'anisotropie est de l'ordre de $\mathrm{Ka}$ : elle est proportionnelle à l'épaisseur de la paroi. La somme de ces deux énergies est minimale pour une certaine valeur $a_{0}$ de $a$ qui est de l'ordre de $\sqrt{A / K}$ tandis que l'énergie superficielle $\gamma$ de paroi est de l'ordre de $2 \sqrt{A K}$.
Pour une couche mince de permalloy orientée par un champ magnétique au cours du dépôt, $A$ est de l'ordre de $10^{-6} \mathrm{erg} / \mathrm{cm}$ et $K$ de l'ordre de $10^{3} \mathrm{erg} / \mathrm{cm}^{3}$, ce qui donne $a_{0}$ de l'ordre de $3 \times 10^{-5} \mathrm{~cm}$ et $\gamma$ de l'ordre de $0,1 \mathrm{crg} / \mathrm{cm}^{2}$.

En réalité, pour que cette évaluation soit correcte, il faut qu'à l'intérieur de la paroi n'apparaisse aucune densité en volume $\rho_{m}$ de charge magnétique; il faut donc que suivant une normale à la paroi la dérivée $\delta J_{\mathrm{n}} / \delta n$, de la projection de $J_{\mathrm{s}}$ sur cette normale, soit nullc. De telles parois sont appelées parois de Bloch (Bloch walls $=\mathrm{BW}$ ). Sur ces bases, on peut développer une théorie précise qui a été bien vérifiée par l'expérience : la mesure directe de l'énergie de paroi a permis de confirmer la valeur théorique avec une précision de quelques pour cent [1].

2. Parois de Bloch (BW) dans les films minces. Dans les films minces, les choses sont différentes. Dès 1955, j'ai fait remarquer [2] qu'à l'intersection de la paroi avec les faces du film apparaissait une densité $\sigma$ de charge superficielle, variant entre $+J_{\mathrm{s}}$ et $-J_{\mathrm{s}}$ (Fig. 1). Il faut donc tenir compte de l'énergie

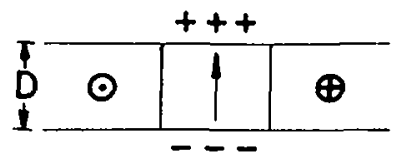

Fig. 1. - Paroi de Bloch dans un film mince. 
magnétostatique correspondante. En l'assimilant à celle d'un cylindre elliptique d'axes principaux $a$ et $D$ (épaisseur du film), l'énergie totale est ainsi

$$
\gamma=\frac{1}{2} \gamma_{0}\left(\frac{a}{a_{0}}+\frac{a_{0}}{a}\right)+\frac{2 \pi a^{2} J_{s}^{2}}{a+D}
$$

qu'il s'agit de minimiser par rapport à $a$. La valeur minimale $\gamma_{\mathrm{B}}$ augmente régulièrement quand $D$ diminue, atteint dans le cas $\mathrm{du}$ permalloy $10 \mathrm{erg} / \mathrm{cm}^{2}$ pour $D=500 \AA \AA$ et $40 \mathrm{erg} / \mathrm{cm}^{2}$ pour $D=10 \AA$; c'est donc un effet considérable. Corrélativement l'épaisseur de paroi diminue et devient inférieure à $100 \AA$ pour $D=10 \AA$.

3. Parois de Néel (NW). - J'ai suggéré [2] que dans les films très minces apparaissait un type spécial de parois, à l'intérieur desquelles l'aimantation variait toujours d'une manière progressive de $+\mathbf{J}_{\mathrm{s}}$ à $-\mathbf{J}_{\mathrm{s}}$, mais en restant parallèle au plan du film (Fig. 2) :

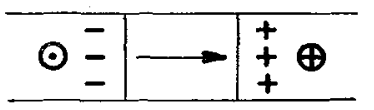

Fig. 2. - Paroi de Néel.

de sorte que les densités superficielles $\sigma$ restent toujours nulles. Par contre apparaissent des charges en volume. Toujours en supposant que l'énergie magnétostatique correspondante est celle d'un cylindre elliptique, l'énergie de paroi s'écrit :

$$
\gamma=\frac{1}{2} \gamma_{0}\left(\frac{a}{a_{0}}+\frac{a_{0}}{a}\right)+\frac{2 \pi a D J_{s}^{2}}{a+D}
$$

En minimisant par rapport à $a$ on trouve une énergie $\gamma_{\mathrm{N}}$ qui croît avec $a$, à partir de $2 \mathrm{erg} / \mathrm{cm}^{2}$ pour $D=10 \AA$ jusqu'à une trentaine $\mathrm{d}^{\prime} \mathrm{erg} / \mathrm{cm}^{2}$ pour les parois très épaisses.

Corrélativement l'épaisseur de paroi décroît de $20000 \AA$ à $200 \AA$.

Ces parois ont reçu le nom de parois de Néel (Néel walls $=\mathrm{NW}$ ).

La comparaison de ces deux résultats montre qu'il existe une certaine épaisseur critique $D_{\mathrm{c}}$ du film, au-dessous de laquelle les parois de moindre énergie sont du type NW, tandis qu'au-dessus, elles sont $\mathrm{du}$ type BW (Fig. 3).

4. Lignes de Bloch. - Aussi bien pour les NW que pour les BW, le vecteur $\mathbf{J}$, pour passer de $+\mathbf{J}_{\mathbf{s}}$ à $-\mathbf{J}_{\mathrm{s}}$, peut tourner soit dans un sens, soit en sens inverse. Ces parois ont des structures identiques au signe près des charges : on peut alors imaginer qu'une

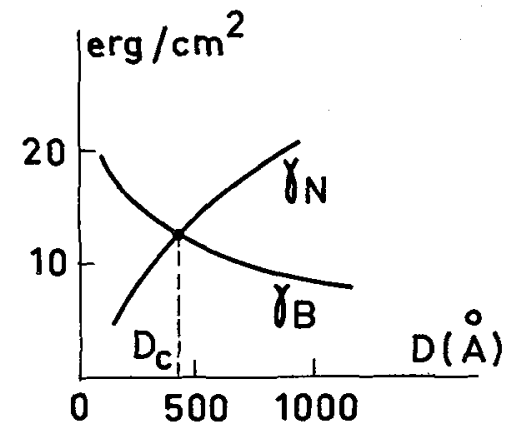

FIG. 3. - Variation schématique de l'énergie des parois de Bloch $\left(\gamma_{B}\right)$ et des parois de Néel $\left(\gamma_{\mathrm{N}}\right)$ en fonction de l'épaisseur $D$ du film.

paroi soit composée d'éléments à sens de rotation positif juxtaposés à des éléments à sens de rotation négatif. Les frontières sont appelées lignes de Bloch. Une telle décomposition diminue l'énergie magnétostatique.

Grâce à l'existence de ces parois d'orientation différente, on a pu montrer expérimentalement que dans un film mince de permalloy au molybdène de $450 \AA$ d'épaisseur, les parois étaient du type NW. En effet lorsqu'on applique un champ magnétique dans le plan du film et normal à la paroi, et qu'on dépose un colloïde ferromagnétique, on observe des segments avec dépôt abondant et des segments avec peu de dépôt. Ce phénomène est prévisible si les parois sont du type NW. Il ne doit pas se produire avec des BW.

5. Les « cross-tie » (CT), - L'expérience montre que dans la région où devrait se produire la transition entre $B W$ et NW, c'est-à-dire pour des épaisseurs de 300 à $1000 \AA$, apparaît une nouvelle structure de paroi très caractéristique, facile à mettre en évidence par la méthode de Bitter ou par microscopie Lorentz. Il s'agit des cross-tie.

La paroi principale est coupée par de petits éléments de paroi perpendiculaire : elle paraît hérissée de barbes, dont la longueur et l'écartement sont de l'ordre du $1 / 100$ de $\mathrm{mm}$.

La microscopie Lorentz permet [3] de déterminer l'orientation de l'aimantation et l'on obtient le schéma de la figure 4, conforme au schéma proposé par Hubert et al. [4].

6. Théorie des cross-tie. - La justification théorique de l'existence des cross-tie demande infiniment plus d'attention que celle des BW ou des NW. On a affaire en effet dans ces derniers cas à des variations énormes d'énergie en fonction de l'épaisseur de la paroi, dans le rapport de 1 à 10,1 à 100 ou davantage, 


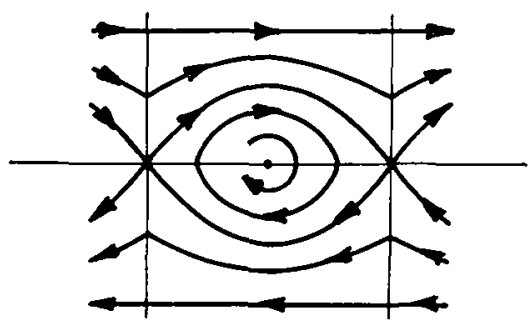

FIG. 4. - Disposition des lignes parallèles

à l'aimantation dans une structure du type «cross-tie ».

de sorte que des erreurs de $50 \%$ dans leur évaluation ne modifient pas les conclusions générales. Il n'en est plus de même maintenant car, dans la région de transition, les énergies des $\mathrm{BW}, \mathrm{NW}$ et des $\mathrm{CT}$ sont très voisines. Il paraît donc indispensable de perfectionner préalablement la théorie des BW et des NW.

Les premières tentatives ont été faites par $\mathrm{S}$. Middelhoek [5]. Dans la suite, W. F. Brown et A. E. LaBonte [6], ont repris le problème d'une manière rigoureuseà l'aide d'une calculatrice digitale, dans le cas d'une dimension, c'est-à-dire en supposant que l'aimantation en un point quelconque ne dépendait que de sa distance au plan médian de paroi : ils décomposent pour cela la paroi en $\mathrm{N}$ prismes uniformément aimantés (par exemple 100 prismes de $30 \AA$ de largeur). Pour une paroi de Bloch ces calculs ont montré que $\beta$ (cosinus directeur de $\mathbf{J}$ correspondant à la normale à la paroi), positif à l'intérieur de la paroi changeait de signe de part et d'autre (Fig. 5), particularité qui n'avait jamais été remarquée jusque-là, quoiqu'au fond assez évidente, puisque de part et d'autre de la paroi, l'aimantation spontanée doit être déviée par le champ de fuite de la partie centrale.

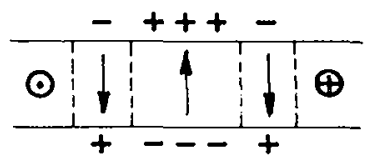

FIG. 5. - Structure schématique réelle d'une paroi de Bloch.

Finalement, les valeurs de $\gamma_{B}$ ainsi calculées sont de 10 à $12 \%$ inférieures à celles de Middelhoek.

Les mêmes méthodes sont applicables aux NW. Tandis que pour les $\mathrm{BW}$, le rapport $a / D$ de l'épaisseur de la paroi à l'épaisseur du film était de l'ordre de l'unité, il peut atteindre une centaine pour les NW, ce qui nécessiterait une décomposition en plus de 1000 prismes, problème inabordable pour la calculatrice. Cette variation de l'aimantation, étalée en une longue queue de part et d'autre du plan médian de la paroi, est due à ce que le champ de fuite de la partie centrale de la paroi favorise cet étalement tandis que c'était le contraire pour une paroi de Bloch.

Un calcul précis n'est pas possible mais il semble finalement que l'épaisseur $D_{\mathrm{c}}$ du film pour laquelle on passe des parois de Néel à celles de Bloch est deux fois plus grande que la valeur initialement estimée par Néel.

Ces conclusions ont été confirmées par un calcul théorique de A. Aharoni [7] qui a montré que $\beta$ variait comme sech $(q x)$. Les énergies obtenues sont de quelques pour cent inférieures à celles obtenues antérieurement par H. D. Dietze et H. Thomas [8] et par Feldtkeller [9].

L'existence d'une longue queue dans la distribution de l'aimantation dans les NW a été mise en évidence expérimentalement par microscopie électronique [9]: [10] dans un film de $450 \AA$ d'épaisseur : l'angle $\theta$ de l'aimantation avec la direction de facile aimantation varie d'abord de $40^{\circ}$ à partir du centre sur $200 \AA$. Les $50^{\circ}$ restants sont étalés sur $3000 \AA$. Corrélativement les champs de dispersion des NW s'étendent beaucoup plus loin que ceux des BW; à égalité de champ, près de 30 fois plus loin, d'après un calcul récent.

7. Energie des cross-tie. - Cette énergie $\gamma_{T}$ est bien plus difficile à calculer que celle des BW ou des NW : $\beta$ n'est plus une fonction de la seule variable $x$, mais des deux variables $x$ et $y$. La tentative la plus intéressante paraît être celle d'Aharoni [11]. Après avoir pris un modèle de distribution d'aimantation conforme aux traits présentés par l'expérience et rappelés plus haut, des calculs compliqués lui fournissent une limite supérieure de l'énergie de $10 \mathrm{erg} / \mathrm{cm}^{2}$ (avec $A=10^{-6} \mathrm{erg} / \mathrm{cm}, J_{\mathrm{s}}=800$ et $K \sim 10^{3} \mathrm{erg} / \mathrm{cm}^{3}$ ).

Cette valeur est plus petite que les valeurs de $\gamma_{\mathrm{B}}$ ou de $\gamma_{N}$ données par les théories les plus anciennes et correspondant à des films d'épaisseur comprise entre 200 et $1000 \AA$ : l'existence des cross-tie serait ainsi justifiée. Malheureusement les théories récentes, plus perfectionnées donnent des valeurs de $\gamma_{B}$ et de $\gamma_{N}$ bien inférieures à $10 \mathrm{erg} / \mathrm{cm}^{2}$. Aharoni attribue la trop grande valeur de sa limite supérieure d'énergie à ce que sa théorie tient compte des contributions des charges en volume pour une épaisseur de film infinie et des contributions des charges superficielles pour une épaisseur zéro. Un calcul ultérieur approché lui montre en effet que la valeur limite supérieure de $\gamma_{\mathrm{CT}}$ pour une épaisseur infinie est de $7,7 \mathrm{erg} / \mathrm{cm}$ et si l'on admet, ce qui est vraisemblable que $\gamma_{C T}$ diminue quand $D$ diminue, on doit en conclure que $7,7 \mathrm{erg} / \mathrm{cm}$ est une vraie limite supérieure pour toute épaisseur. 
Cette limite est encore plus élevée que les valeurs exactes de $\gamma_{B}\left(4,5 \mathrm{erg} / \mathrm{cm}^{2}\right.$ pour $D=500 \AA$, épaisseur pour laquelle $\gamma_{\mathrm{B}}=\gamma_{\mathrm{N}}$ ).

Il faut sans doute en conclure que la distribution de l'aimantation utilisée par Aharoni n'est pas celle qui correspond à l'énergie minimale. Le problème de l'énergie des CT reste donc à résoudre. Il s'agit d'ailleurs de difficultés communes à tous les problèmes de micromagnétisme; la nature trouve bien plus facilement que les théoriciens les distributions d'énergie minimale.

8. Films mono- et polycristallins. - Dans tous les calculs qui précèdent, on suppose implicitement que le film est homogène, c'est-à-dire monocristallin. En réalité, nous avons affaire à un polycristal dont les cristallites sont orientés au hasard et ont des dimensions très petites, souvent de 50 à $100 \AA$ de diamètre.

En ce qui concerne l'énergie magnétocristalline, ceci est sans grande conséquence car il s'agit d'une énergie induite par le champ magnétique qui a agi au cours du dépôt et qui ne dépend peut-être pas beaucoup de l'orientation des cristallites : de toutes façons on peut prendre un certain $K$ moyen.

En ce qui concerne l'énergie magnétostatique, ceci est aussi sans grande importance : dans chaque cristallite l'aimantation s'écarte un peu de la direction moyenne, ce qui se traduit par des ondulations (ripples) de l'aimantation, mais ne change guère la valeur de l'énergie magnétostatique.

Il peut en être tout autrement pour l'énergie d'échange. Tout dépend de sa valeur entre atomes proches voisins situés de part et d'autre de la frontière entre deux grains. Nous ne connaissons absolument rien à ce sujet. Il se peut que cette énergie soit du même ordre de grandeur que s'il y avait continuité cristalline. Il se peut aussi que cette énergie soit nulle, c'est-à-dire que les moments magnétiques de deux cristallites contigus ne soient pas couplés par échange. Dans le premier cas on doit s'attendre à une variation du coefficient $A$ de quelques pour cent. Dans le second, les répercussions sont bien plus considérables.

Considérons en effet une répartition schématique de cristallites cubiques de côté $b$ représentés par la figure 6 ct rapportée à deux axes rectangulaires ox et oy. $\mathrm{Au}$ voisinage du point $\mathrm{O}$, s'il y avait continuité magnétique, les cosinus directeurs de l'aimantation s'écriraient en négligeant les termes du deuxième ordre :

$$
\alpha=0 ; \beta=k x ; \gamma=1 \text {. }
$$

La densité d'énergie d'échange serait ainsi :

$$
F_{\mathrm{w}}=A(\nabla x)^{2}=A k^{2} \text {. }
$$

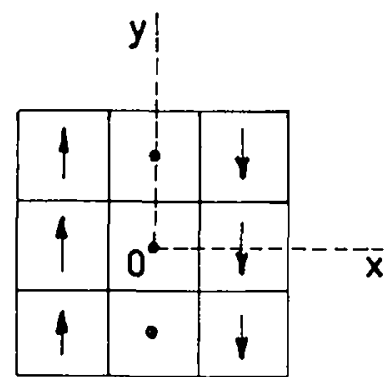

FIG. 6.

$\mathrm{Au}$ contraire, si les cristallites sont indépendants magnétiquement, l'énergie minimale correspond en première approximation à une aimantation uniforme de chaque cristallite, égale à l'aimantation au centre de chaque cristallite, donnée par les équations (3). On a alors $F_{\mathrm{w}}=0$. Tout se passe comme si $A$ était nul. Mais on peut aussi imaginer une répartition des cristallites en quinconce (Fig. 7). Les faces des cristallites perpendiculaires à oy portent des charges superficielles égales à $\pm J_{s} k b / 2$.

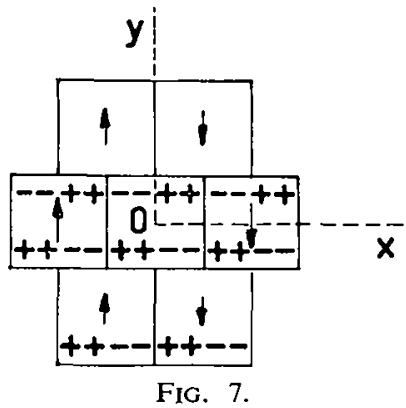

L'énergie correspondante par $\mathrm{cm}^{3}$ est de l'ordre de $J_{\mathrm{s}}^{2} b^{2} k^{2} / 10$. Comme nous avons un mélange des deux configurations, tout se passe comme si nous avions une constante d'échange de l'ordre de :

$$
A^{*} \sim J_{s}^{2} b^{2} / 20 \text {. }
$$

Pour des grains de diamètre $b=50 \AA$, on aurait ainsi

$$
A^{*} \sim 10^{-8} \mathrm{erg} / \mathrm{cm} .
$$

$A^{*}$ serait ainsi cent fois plus petit que $A$. Dans de telles parois polycristallines, les épaisseurs de parois devraient être ainsi dix fois plus grandes que pour les lames monocristallines.

La théorie qui vient d'être esquissée est naturellement très schématique : elle n'est valable que lorsque $A^{*}$ est bien plus petit que $A$. Quand ces deux quantités sont du même ordre de grandeur, il faut tenir compte 
de la variation de la direction de l'aimantation spontanée à l'intérieur d'un même cristallite.

Les quelques données expérimentales relatives aux déterminations de largeur de paroi par microscopie Lorentz semblent donner des valeurs de $A$ normales, compatibles avec les valeurs fournies par la constante de Curie et la température de Curie. De même les valeurs de $A$ données par les spectres de résonance des ondes de spin [12] dans des films de permalloy de $3900 \AA$ d'épaisseur sont également voisines des valeurs attendues classiquement.

Il faut en conclure qu'il existe une continuité magnétique d'échange entre grains contigus. $\mathrm{Au}$ fond ce résultat peut paraître normal si l'on se représente l'état des atomes dans les joints intergranulaires comme une sorte d'état liquide. Or on sait que dans les métaux ferromagnétiques le passage de l'état cristallisé à l'état liquide modifie très peu les propriétés paramagnétiques et notamment le champ moléculaire.

De toutes façons il conviendrait de reprendre des déterminations soignées de l'épaisseur des parois par microscopie Lorentz, pour des valeurs très différentes des dimensions des cristallites et pour la même valeur de l'épaisseur du film. Il est possible que certaines conditions de dépôt fournissent des cristallites indépendants.

9. Action d'un champ normal à la paroi. - En appliquant un champ magnétique, dans le plan du film normalement à la paroi, les directions des aimantations spontanées des deux domaines adjacents se rapprochent et font entre elles un angle $2 \varphi$ qui varie de $\pi$ à 0 quand $H$ varie de 0 à $2 K / J_{\mathrm{s}}$.

Pour $D=500 \AA$, les valeurs de $\gamma_{\mathrm{B}}$ et $\gamma_{\mathrm{N}}$ varient [13] avec $\varphi$ comme l'indique schématiquement la figure 8 ;

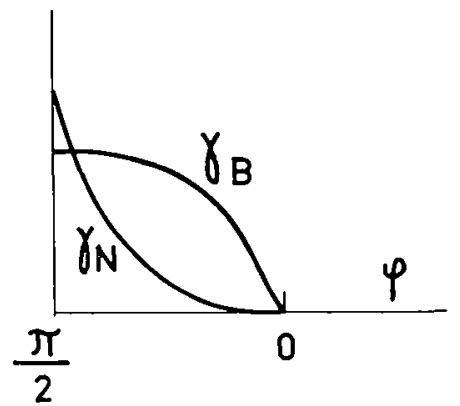

FIG. 8. - Variation comparée des énergies des parois de Bloch et de Néel en fonction de l'angle $2 \varphi$ des aimantations spontanées des deux domaines adjacents.

$\gamma_{N}$ tend vers zéro beaucoup plus rapidement que $\gamma_{B}$. La valeur critique $D_{c}$ d'épaisseur de film correspon- dant à la transition entre les deux types de parois augmente donc quánd $\varphi$ tend vers zéro. On doit donc, si $\varphi$ est suffisamment petit, observer des NW dans des matériaux massifs. Gemperle et Zelený [14] ont effectivement observé des NW dans des lames minces de cobalt et de magnétoplumbite de quelques dixièmes de millimètre d'épaisseur : il faut pour cela que la valeur réduite du champ

$$
h=H_{0} J_{\mathrm{s}} / 2 \mathrm{~K}
$$

soit suffisamment voisine de l'unité ( 0,8 par exemple). Ces parois s'orientent d'une manière stable normalement au champ ce qui montre bien que ce sont des NW. L'énergie d'une BW est en effet plus grande lorsqu'elle est normale au champ que lorsqu'elle est parallèle. Au surplus, dans de telles conditions, pour des parois perpendiculaires au champ, l'énergie des NW est beaucoup plus faible que celle des BW.

Il est d'ailleurs possible que pour des valeurs de $\varphi$ inférieures à $\pi / 2$, les parois soient d'un type mixte, intermédiaire entre NW et BW. La figure 9 due à Torok, Olson et Oredson [15] indique le pourcentage

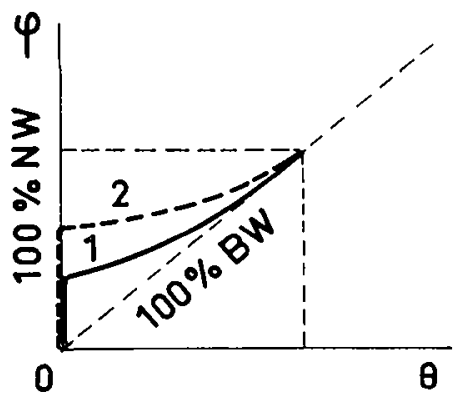

Fig. 9. - Variation, en fonction de l'angle $2 \varphi$ (angle des aimantations spontanćes des deux domaines adjacents), de l'angle $\theta$ (angle avec la normale à la paroi de l'aimantation correspondant au plan médian de la paroi).

d'hybridation en fonction de $\varphi$ et de 0 angle de l'aimantation spontanée avec la direction du champ dans le plan médian (pour une paroi de Bloch $\theta=\pi / 2$, pour une $\mathrm{NW}, \theta=0$ ).

10. Parois et quasi-parois dans les films doubles. Jusqu'ici nous avons étudié ce qui se passe dans un film ferromagnétique simple. Mais on s'est aperçu qu'en déposant l'un au-dessus de l'autre deux films minces ferromagnétiques, séparés par un film mince non magnétique, par exemple un film de monoxyde de silicium $\mathrm{SiO}$, on obtenait des modifications importantes des propriétés magnétiques. Pour simplifier et à moins d'indication contraire, nous considérerons deux films ferromagnétiques de même épaisseur $D$ 
et nous désignons par $b$ l'épaisseur du film intermédiaire.

Supposons qu'une paroi de Néel soit située dans l'un des films : elle est entourée d'un champ de dispersion important qui induit des variations de l'aimantation du second film ferromagnétique. On peut ainsi imaginer avec Middelhoek [16] que la paroi de Néel de l'un des films (Fig. 10a) surmontée d'une quasiparoi (Fig. 10b) constituée par la juxtaposition de

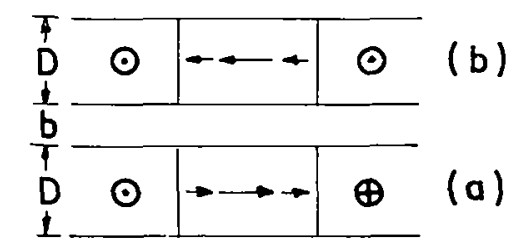

Fig. 10. - Paroi et quasi-paroi de Néel.

deux demi-parois de Néel, à sens de rotation opposé, de sorte que de part et d'autre de la quasi-paroi, les aimantations spontanées sont dirigées dans le même sens. La répartition des charges magnétiques dans une quasi-paroi est la même que dans une paroi. En associant une paroi à une quasi-paroi de polarité opposée, on diminue ainsi l'énergie magnétostatique d'une manière considérable.

Middelhoek [17] calcule une énergie $\gamma_{\mathrm{p}}$ de paroi, rapportée à $1 \mathrm{~cm}^{2}$ :

$$
\left.\gamma_{\mathrm{p}}=\pi \sqrt{2 K\left[A+\pi J_{\mathrm{s}}^{2}\right.} D\left(\frac{1}{2} b+\frac{1}{3} D\right)\right] .
$$

Un autre auteur trouve une expression analogue [18].

La valeur de $2 \gamma_{p}$ est beaucoup plus faible que celle de $\gamma_{B}, \gamma_{N}$ ou $\gamma_{C T}$, comme le montre la figurc 11, relative à $b=100 \AA$.

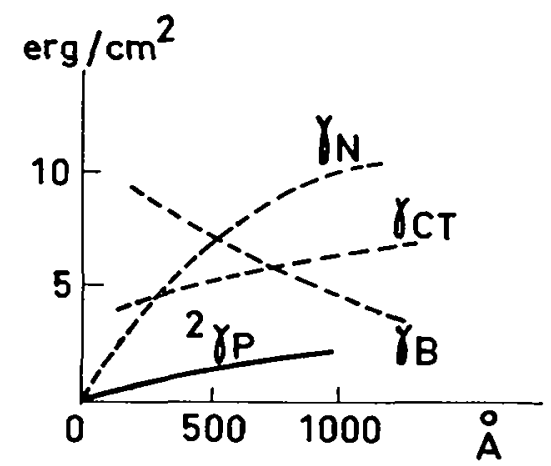

FiG. 11. - Variation schématique avec l'épaisseur du film de l'éncrgie de différents types de paroi $\left(\gamma_{B}, \gamma_{\alpha}, \gamma_{C T}\right)$ et d'une paroi de Néel associée à une quasi-paroi $\left(2 \gamma_{p}\right)$.

Slonczewski a montré [19] au surplus que la valeur réelle de $\gamma_{\mathrm{p}}$ était encore plus faible que celle qui a été indiquée plus haut, car l'aimantation s'écarte en réalité légèrement $d u$ plan des films si bien que l'énergie magnétostatique diminue $: \gamma_{\mathrm{p}}$ peut ainsi être encore réduit de moitié.

Il en résulte que dans les films multiples, le domaine d'existence des NW associées à des QNW s'étend beaucoup aux dépens de celui des BW comme le montre la figure 12 .

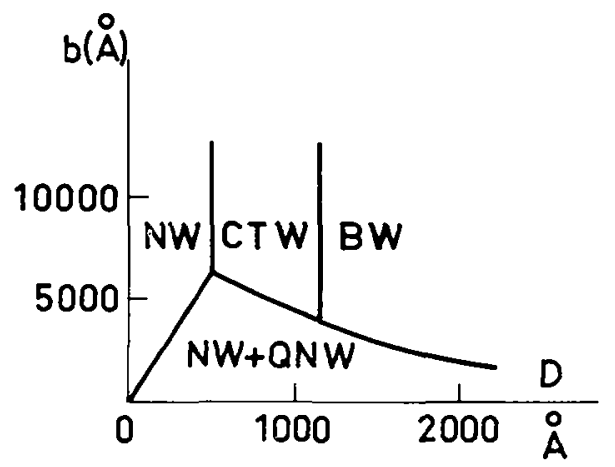

Fig. 12. - Domaine d'existence des différents types de paroi dans un film double ( $D$ : : épaisseur des films; $b=$ leur distance).

L'expérience montre en effet que deux films minces qui montrent des cross-tie lorsqu'ils sont séparés l'un de l'autre, montrent des NW lorsqu'on les dispose à proximité l'un de l'autre.

11. Existence possible de quasi-parois de Bloch (QBW). - On constate aisément que l'association d'une BW avec une QBW (Fig. 13), conduit à neutrali-

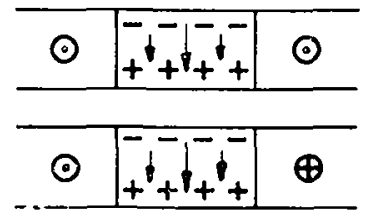

FIG. 13. - Paroi et quasi-paroi de Bloch.

ser les charges superficielles disposées en regard l'une de l'autre, de sorte que au mieux tout se passe comme s'il s'agissait d'une paroi BW d'épaisseur $2 \mathrm{D}$. Comme l'énergie des BW varie assez peu avec $D$, on en conclut que l'association de $\mathrm{BW}$ à des QBW ne diminue pas sensiblement l'énergic. Lcs associations $\mathrm{NW}+\mathrm{QNW}$ restent donc énergétiquement beaucoup plus favorables.

12. Rôle des épaisseurs relatives des deux films ferromagnétiques d'un film multiple. - Les considérations développées plus haut se rapportent à des films ferromagnétiques couplés, de même épaisscur $D$. 
Il est bien évident que si le film dans lequel se développe la QNW est plus épais que le film contenant la vraie NW, la fermeture du flux sera facilitée et l'énergie de paroi diminuera. Ce sera l'inverse si le film contenant la QNW est moins épais que l'autre.

13. Visibilité des QNW. - Les QNW doivent être difficiles à mettre en évidence par la méthode de Bitter, car la densité des charges magnétiques, volumiques ou superficielles, y est essentiellement faible. La situation change lorsqu'on applique, parallèlement au plan du film multiple, un champ magnétique dirigé au sens inverse de l'aimantation existant au milieu de la NW : il en résulte une augmentation des densités volumiques de charge de la NW. Si l'épaisseur des deux films ferromagnétiques est la même, la QNW ne peut trouver une configuration susceptible d'annuler les charges de la NW : des charges volumiques subsistent et la QNW devient visible par la méthode de Bitter.

14. Association de deux parois de Néel dans un film double. - La même diminution spectaculaire de l'énergie de paroi se produit lorsqu'on associe dans les deux films deux vraies NW au lieu d'associer une NW à une QNW : les calculs sont à peu près identiques. Comme l'a remarqué Slonczewski [20], les deux vraies NW, situées dans les deux films superposés, peuvent être telles que les aimantations spontanées des deux domaines superposés soient parallèles (doubles parois de première espèce, Fig. 14a) ou telles que les domaines superposés possèdent des aimantations spontanées antiparallèles (doubles parois de deuxième espèce, Fig. 14b). Il nc semble pas que jusqu'ici de telles doubles parois aient été observées. Les doubles parois de deuxième espèce doivent être à peu près insensibles à l'action d'un champ magnétique.

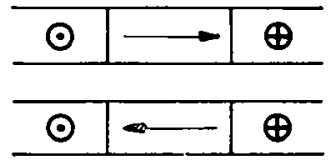

(a)

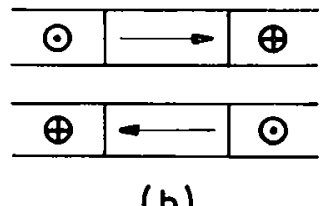

(b)
FIG. 14. - Deux NW dans un film double.

15. Paires de parois. - Des associations de deux parois, voisines et parallèles, ont été observées [21] dans des films très minces de cobalt $(75 \AA)$ ou de permalloy au molybdène $(147 \AA)$. Ces paires de parois s'observaient après désaimantation dans un champ alternatif parallèle à la direction de difficile aimantation.
On peut imaginer pour les BW les deux catégories de paires de la figure 15 : en (a), les aimantations tournent en sens inverse dans les deux parois; en (b), elles tournent dans le même sens. En (a), les deux

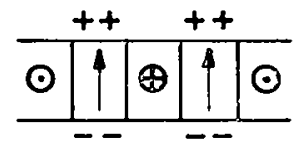

(c)

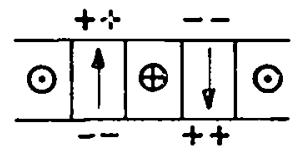

(b)
Fig. 15. - Paires de parois de Bloch dans un film simple.

parois se repoussent jusqu'à une certaine distance critique au-dessous de laquelle la double paroi s'évanouit, comme le montre un calcul rigoureux [22]. En (b) les deux parois s'attirent et atteignent en champ nul une distance d'équilibre.

Pour des NW, on peut imaginer des configurations analogues avec en (a) des aimantations qui tournent en sens inverse dans les deux parois et en (b) des aimantations qui tournent dans le même sens (Fig. 16).

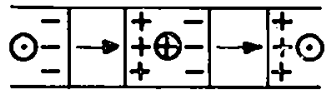

(a)

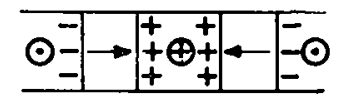

(b)
FIG. 16. - Paires de parois de Néel dans un film simple.

Dans la configuration (b) les deux parois se repoussent de sorte qu'une paire de telles parois ne peut subsister en l'absence de champ appliqué. Dans la configuration (a) les deux parois s'attirent et rien n'empêche les deux parois de s'annihiler par rotation de l'aimantation du domaine situé entre les deux parois : une rotation convenable de l'aimantation du film, dans la région située entre les deux parois diminue à la fois l'énergie magnétostatique et l'énergie d'échange.

Le comportement des doubles parois observées expérimentalement et le fait qu'un champ normal au plan du film provoque un dépôt plus dense sur l'une des parois de la paire suggère que dans les observations citées plus haut il s'agit de BW du type (b). On nc comprend pas très bien alors pourquoi on a affaire à des BW plutôt qu'à des NW dans des films aussi minces. On serait tenté d'attribuer cette particularité à l'existence d'une anisotropie perpendiculaire. Rappelons à ce propos que les expériences de Fujiwara et al. [23] ont révélé des anisotropies perpendiculaires, avec des valeurs de $K$ comprises entre 0 et $-2 \times 10^{6}$ 
$\mathrm{erg} / \mathrm{cm}^{3}$, variant ainsi beaucoup suivant les conditions de dépôt du permalloy.

Des difficultés d'interprétation subsistent donc encore.

16. Conclusions. - En résumé, on peut conclure que seul le problème des parois simples de Bloch dans les films minces est bien résolu. Il subsiste encore des incertitudes sur la structure et l'énergie exactes des parois de Néel. Quant aux structures en icrosstie ), l'évaluation de leur énergie reste assez grossière, en l'absence de méthode théorique satisfaisante pour traiter le problème. Naturellement ces difficultés se retrouvent augmentées dans tous les problèmes d'association de parois et de quasi-parois.

On peut d'ailleuis se demander si ces difficultés sont simplement d'ordre mathématique dans le traitement d'un film à faces parallèles défini par les trois constantes $A, K$ et $J_{\mathrm{s}}$. En réalité le film n'est pas homogène, il existe des cristallites qui ne sont peut-être pas jointifs, les faces du film ne sont pas planes, etc... Il y a peut-être à tenir compte d'une anisotropie perpendiculaire.

Il subsiste donc de nombreux problèmes à résoudre, dont la plupart semblent être d'ordre expérimental et liés à une maîtrise totale des conditions de dépôt.

\section{Bibliographie}

[1] Brissonneau et Néel, C. R. Acad. Sc., 1962, 254, pp. 2891-2896; J. Appl. Phys., 1963, 34, 4, p. 1321.

[2] Néel (L.), C. R. Acad. Sc., 241, 1955, p. 533.

[3] Fuchs, Naturwiss., 1960, 47, 392.

[4] Huber, Smith et Goodenough, J. Appl. Physics, 1958, 29, 294.

[5] MidDELHOEK (S.), Thèse, Univ. d'Amsterdam, 1961. J. Appl. Physics, 1963, 34, 1054.

[6] Brown (W. F.) et LaBonte, J. Appl. Physics, 1965, $36,1380$.

[7] Aharoni (A.), J. Appl. Physics, 1966, 37, 3271.
[8] Z. Physik, 1961, 163, 523.

[9] Feldtkeller (E.), Z. Angew. Physics, 1963, 15, 206.

[10] Fuchs (E.), Naturwiss., 1960, 47, 392.

[11] Aharoni (A.), J. Appl. Physics, 1966, 37, 4615.

[12] Seavey Jr (M. H.) et Tannenwald (P. E.), Phys. Rev. Letters, 1958, 1, 168; J. Appl. Phys., 1959, 30, $227 \mathrm{~S}$.

[13] Middelhoek, Thèse, Amsterdam, 1961.

[14] Gemperle (R.) et Zelený (M.), Phys. Stat. Sol., 1964, 6, 839 .

[15] J. Appl. Phys., 1965, 36, 1394.

[16] Middelhoek (S.), Z. Angew. Phys., 1965, 18, 524.

[17] Slonczewski (J. C.) et Middelhoek (S.), Appl. Phys. Letters, 1965, 6, 139.

[18] Feldtkelder (E.), Z. Angew. Phys., 1965, 18, 532.

[19] Slonczewski (J. C.), J. Appl. Phys., 1966, 37, 1268 ; I.B.M. J. Res. Dev., 1966, 10, 377.

[20] IBM, J. Res. Dev., 1966, 10, 377.

[21] Winliams et Sherwood, J. Appl. Phys., 1957, $28,548$.

[22] LaBonte (A. E.) et Brown (W. F.) Jr, J. Appl. Phys., 1966, 37, 1299.

[23] Fujiwara (S.), Koikeda (T.), Chikazumi (S.), J. Phys. Soc. Japan, $878 ; 20,1694$, KoIKEDA (T.) et Chrkazumi (S.), J. Phys. Soc. Japan, 1966, 21, 399.

\section{DISCUSSION}

M. Kléman. - Les quasi-parois de Néel, considérées isolément, ayant la même énergie que les parois de Néel, peut-on les observer sur des lames uniques?

M. NéEL. - Non, les quasi-parois sont excitées par les parois de Néel et ne peuvent exister que s'il existe deux couches ferromagnétiques, essentiellement séparées par un petit intervalle non magnétique. Une quasi-paroi isolée, supposée exister à un moment donné, doit s'évanouir spontanément en un intervalle de temps extrêmement faible.

M. WADE. - Je crois que les structures de grains séparés que vous avez proposées sont réalisables. expérimentalement par évaporation sous vide. Ceci peut donner un intérêt expérimental à vos propos. 